\title{
Orthogonal art galleries with holes: a coloring proof of Aggarwal's Theorem
}

\author{
Paweł Żyliński* \\ Institute of Mathematics \\ University of Gdańsk, 80952 Gdańsk, Poland \\ pz@math.univ.gda.pl
}

Submitted: Sep 29, 2005; Accepted: Feb 27, 2006; Published: Mar 7, 2006

Mathematics Subject Classifications: 05C15, 05C90

\begin{abstract}
We prove that $\left\lfloor\frac{n+h}{4}\right\rfloor$ vertex guards are always sufficient to see the entire interior of an $n$-vertex orthogonal polygon $P$ with an arbitrary number $h$ of holes provided that there exists a quadrilateralization whose dual graph is a cactus. Our proof is based upon 4-coloring of a quadrilateralization graph, and it is similar to that of Kahn and others for orthogonal polygons without holes. Consequently, we provide an alternate proof of Aggarwal's theorem asserting that $\left\lfloor\frac{n+h}{4}\right\rfloor$ vertex guards always suffice to cover any $n$-vertex orthogonal polygon with $h \leq 2$ holes.
\end{abstract}

\section{Introduction}

The art gallery problem was originally posed by Victor Klee in 1973 as the question of determining the minimum number of guards sufficient to see every point of the interior of an $n$-vertex simple polygon. The guard was assumed to be a stationary point which can see any point that can be connected to it with a line segment within the polygon. The first result was due to Chvátal [2] who proved that $\left\lfloor\frac{n}{3}\right\rfloor$ guards are sometimes necessary and always sufficient to cover a polygon with $n$ vertices. His entirely combinatorial proof started with an arbitrary triangulation of the polygon and then cut off a small piece for the inductive step. Three years later, in 1978, Fisk [3] offered a simpler proof of Chvátal's result based upon a 3-coloring of the triangulation graph. Since then many different variations of this problem have arisen; see [7], [8] for more details.

An orthogonal gallery is a polygon whose edges are either horizontal or vertical, see Fig. 1(a). An orthogonal gallery with holes is an orthogonal polygon $P$ enclosing some other orthogonal polygons $H_{1}, \ldots, H_{h}$, known as the holes. None of the boundaries of

${ }^{*}$ Supported by the State Committee for Scientific Research (Poland) Grant No. 4 T11C 04725. 
(a)

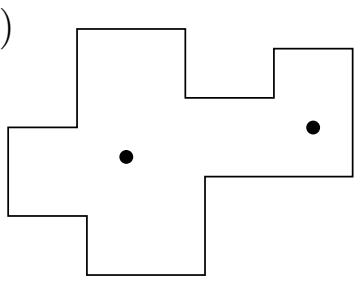

(b)

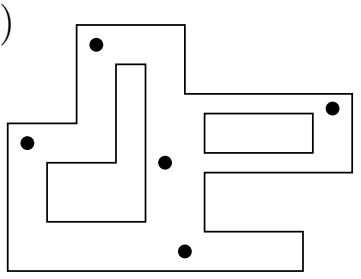

(c)

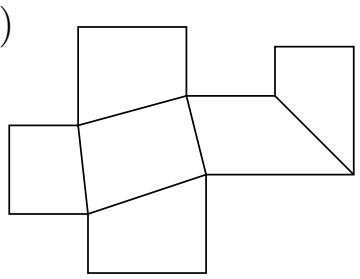

Figure 1: (a) An orthogonal polygon $P$ that requires 2 guards (black dots). (b) An orthogonal polygon with two holes that requires 5 guards. (c) A quadrilateralization of $P$.

polygons $P, H_{1}, \ldots, H_{h}$ may intersect, and each of the holes is empty. (See Fig. 1(b).) The first major result concerning orthogonal galleries was presented by Kahn, Klawe and Kleitman [6]. They proved that a hole-free orthogonal polygon with $n$ vertices can always be guarded by $\left\lfloor\frac{n}{4}\right\rfloor$ guards even if guards are restricted to locations at vertices of the polygon only - so-called vertex guards. Their proof is based upon quadrilateralization and a 4-coloring of the resultant quadrilateralization graph. Let us recall that a quadrilateralization of an orthogonal polygon $P$ (with or without holes) is a partitioning of $P$ into a set of convex quadrilaterals with pairwise disjoint interiors in such a way that the edges of those quadrilaterals are either edges or internal diagonals of $P$ joining pairs of vertices - see Fig 1(c); the existence of a quadrilateralization was established by Kahn et al. [6]. In 1982, O'Rourke [7] conjectured the $\left\lfloor\frac{n}{4}\right\rfloor$-bound to be the sufficiency bound for orthogonal polygons with holes as well, and this conjecture was verified by Hoffmann [4]. However, when speaking about vertex guards, the Art Gallery Problem in orthogonal galleries with holes generally remains open. One of the oldest conjectures concerning guarding polygons with holes is due to Shermer (see Fig. 2):

Conjecture 1.1 (Shermer's Conjecture 1982) Any orthogonal polygon with $n$ vertices and $h$ holes can always be guarded by $\left\lfloor\frac{n+h}{4}\right\rfloor$ vertex guards.

Of course, this conjecture is true for $h=0$ by the result of Kahn et al. [6]. In 1984, Aggarwal [1] showed the validity of Shermer's conjecture for orthogonal galleries with at most 2 holes. His proof, occupying several pages, is based upon a reduced quadrilateralization of a polygon.

Theorem 1.2 (Aggarwal 1984) Shermer's Conjecture is true for $h=1,2$.

The best currently known upper bounds for vertex guards in orthogonal polygons with an arbitrary number of holes are the following:

Theorem 1.3 (O'Rourke 1987) An orthogonal polygon with $n$ vertices and $h$ holes can always be guarded by $\left\lfloor\frac{n+2 h}{4}\right\rfloor$ vertex guards.

Theorem 1.4 (Hoffmann, Kriegel 1996) An n-vertex orthogonal polygon with holes can always be guarded by $\left\lfloor\frac{n}{3}\right\rfloor$ vertex guards. 


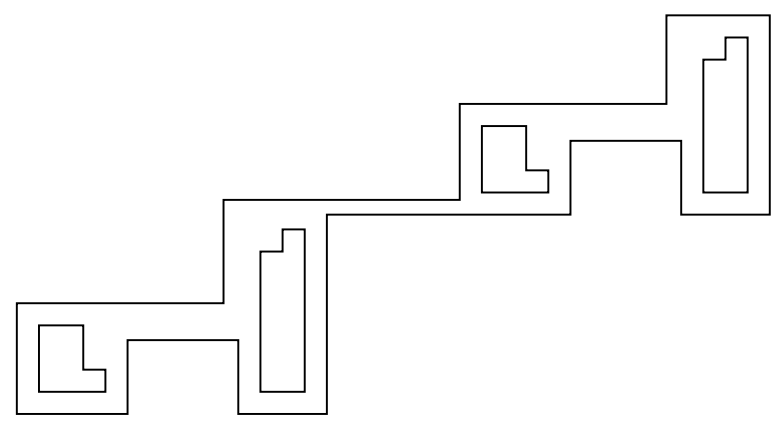

Figure 2: An orthogonal polygon with holes that requires $\left\lfloor\frac{n+h}{4}\right\rfloor$ vertex guards; here $n=44, h=4$ and the polygon requires 12 vertex guards. When not limited to vertices, 11 guards are sufficient.

The upper bound of the preceding theorem is better than O'Rourke's bound of $\lfloor(n+2 h) / 4\rfloor$ when the number of holes $h$ is large. This is because $n$, the total number of vertices, includes the vertices of the holes.

Our result. Let us recall that a connected graph satisfying the property that any two of its cycles share at most one vertex and any such vertex is a cut-vertex, is called a cactus. In this paper, our main result is the following theorem:

Theorem 1.5 Let $P$ be an $n$-vertex orthogonal polygon with $h$ holes, and suppose there exists a quadrilateralization of $P$ whose dual graph is a cactus. Then $\left\lfloor\frac{n+h}{4}\right\rfloor$ vertex guards are always sufficient to see the entire interior of $P$.

It is worth pointing out that this result provides an alternate short proof of Aggarwal's theorem, as we shall discuss in Subsection 2.1. The proof of Theorem 1.5 is similar in spirit to that in [6]. We consider a graph obtained from a quadrilateralization of any orthogonal gallery with one hole. We show this graph to be 4-colorable, although sometimes we have to cut the gallery by splitting some vertex into two vertices. Next, the positions of guards are determined by vertices of a color with the minimum number of occurrences, i.e not more than $\left\lfloor\frac{n+1}{4}\right\rfloor$ times. Then a simple extension of this fact leads to Theorem 1.5, as we discuss in Section 3.

\section{Orthogonal galleries with one hole}

Let $Q$ be a quadrilateralization of an $n$-vertex orthogonal polygon $P$ with one hole. By adding both diagonals to each quadrilateral of $Q$, we get the quadrilateralization graph $G_{Q}$ whose vertices correspond to the vertices of the polygon and whose edges correspond to the edges of polygon $P$, to the diagonals of quadrilateralization $Q$, and to the added internal diagonals of all quadrilaterals, as depicted in Fig. 3. Our goal is to 4-color graph $G_{Q}$. 
(a)

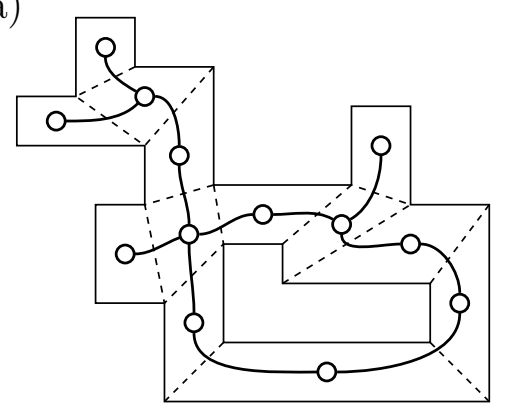

(b)

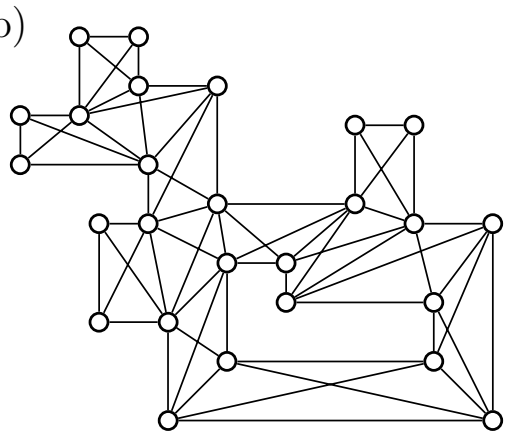

Figure 3: (a) An orthogonal polygon $P$ with one hole, its quadrilateralization, and the dual graph $G_{D}$. (b) Quadrilateralization graph $G_{Q}$.

(a)

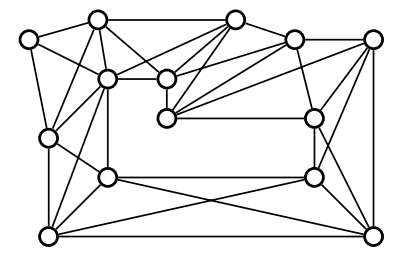

(b)

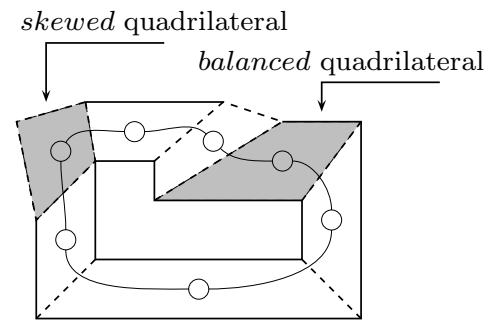

Figure 4: (a) Graph $G_{Q}^{k}$. (b) Balanced and skewed quadrilaterals.

Let $G_{D}$ be the dual graph of quadrilateralization $Q$ : each vertex of $G_{D}$ corresponds to a quadrilateral and two vertices are adjacent if their quadrilaterals share a side. Clearly, the dual graph $G_{D}$ is a single cycle with some number of attached trees. Consider now any leaf $q$ of dual graph $G_{D}$ and its corresponding quadrilateral in the quadrilateralization graph $G_{Q}$. Two of $q$ 's vertices are of degree 3 , so we can remove them, thus getting a graph $G_{Q}^{1}$. Of course, if $G_{Q}^{1}$ is 4-colorable, then so is $G_{Q}$. Proceeding in a similar recursive manner with all leaves, we eventually get a graph $G_{Q}^{k}$ that consists only of quadrilaterals (with diagonals) that form a single cycle $\mathrm{C}$ in the dual graph $G_{Q}$. (See Fig. 4.) It is obvious that $G_{Q}$ is 4-colorable if and only if $G_{Q}^{k}$ is 4-colorable.

Let us recall a simple characterization of the cycle quadrilaterals [1]. Each of the cycle quadrilaterals has all four of its vertices on the boundary of the polygon formed by cycle $C$. If a quadrilateral has two vertices on the exterior boundary and two on the boundary of the hole, it is called balanced, otherwise it is called skewed.

Lemma 2.1 [1] The cycle in the dual graph of any quadrilateralization of an orthogonal polygon with one hole has an even number (at least four) of balanced quadrilaterals.

The next step is to observe that each skewed quadrilateral has one vertex of degree 3 in graph $G_{Q}^{k}$. Removing all such vertices from all skewed quadrilaterals results in a graph $G_{Q}^{*}$ that consists of an even number of balanced quadrilaterals (with their diagonals) and a certain number of triangles. The following claim is obvious. 
(a)

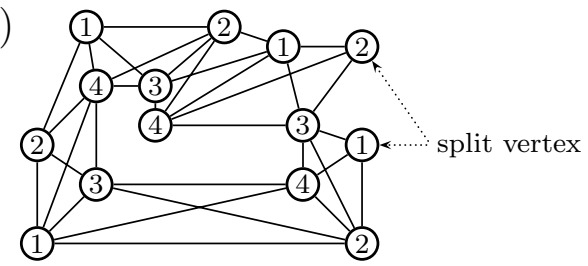

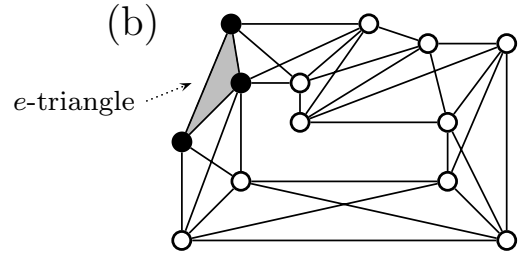

Figure 5: (a) Sometimes we have to split a vertex into two. (b) An e-triangle.

Claim 2.2 $G_{Q}$ is 4-colorable if and only if $G_{Q}^{*}$ is 4-colorable.

Thus all that remains is the proof that $G_{Q}^{*}$ is 4-colorable. But, as one could expect, this does not always hold: if we consider a graph $G_{Q}^{*}$ that results from removing the vertex of degree 3 from the graph $G_{Q}^{k}$ shown in Fig. 4(a), then it is easy to check that $G_{Q}^{*}$ is not 4-colorable. However, by splitting any vertex that belongs to the external face of $G_{Q}^{*}$ into two vertices, we get a 4-colorable graph, see Fig. 5(a). We will show that similar reasoning can be applied to any graph $G_{Q}^{*}$. Of course, this will lead to a weaker result than 4-colorability of $G_{Q}^{*}$, but this will suffice for our purpose of showing the upper $\left\lfloor\frac{n+1}{4}\right\rfloor$-bound.

Lemma 2.3 For any quadrilateralization $Q$ of an orthogonal polygon with one hole, the graph $G_{Q}^{*}$ is 4-colorable, although sometimes we have to split a vertex into two in order to get a legal 4-coloring.

Proof. In $G_{Q}^{*}$ we define the internal cycle as the cycle of $G_{Q}^{*}$ all of whose vertices are vertices of the hole; clearly, such a cycle is unique. Similarly, the external cycle is the (unique) cycle of $G_{Q}^{*}$ none of whose vertices is a vertex of the hole. Thus in graph $G_{Q}^{*}$ we can distinguish two kinds of triangles resulting from skewed quadrilaterals whose degree 3 vertices were deleted: so-called $e$-triangles and $i$-triangles. An $e$-triangle (resp. $i$-triangle) is the triangle of $G_{Q}^{*}$ that results from a skewed quadrilateral and has two vertices on the external (resp. internal) cycle of $G_{Q}^{*}$, as in Fig. 5(b). Now, the proof will proceed in four cases, depending on the number of $e$-triangles and $i$-triangles.

Case 1: The number of $i$-triangles is even, and the number of e-triangles is even in $G_{Q}^{*}$. By Lemma 2.1, $G_{Q}^{*}$ has $m=2 l$ vertices, $l \geq 4$, and we can label the vertices on the external cycle with $v_{1}, v_{2}, \ldots, v_{2 k}$, for some $k \geq 2$, and the vertices on the internal cycle with $v_{2 k+1}, \ldots, v_{m}$, in the clockwise manner. (See Fig. 6.) Then it is easy to see that the function $c$ defined below is a legal 4-coloring of graph $G_{Q}^{*}$.

$$
c\left(v_{i}\right)= \begin{cases}1 & \text { if }(i \leq 2 k) \text { and }(i \bmod 2=1), \\ 2 & \text { if }(i \leq 2 k) \text { and }(i \bmod 2=0), \\ 3 & \text { if }(2 k<i \leq 2 l=m) \text { and }(i \bmod 2=1) \\ 4 & \text { if }(2 k<i \leq 2 l=m) \text { and }(i \bmod 2=0)\end{cases}
$$

In all the following cases, to 4-color graph $G_{Q}^{*}$ we will have to split exactly one vertex into two vertices. 
(a)

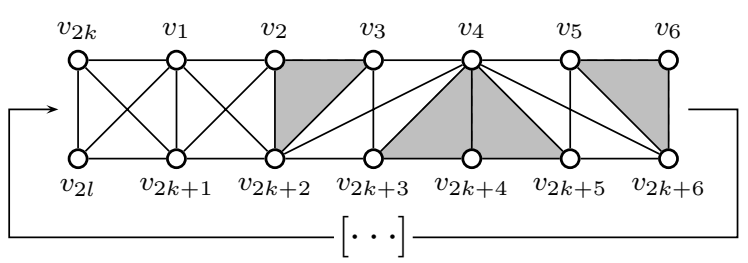

(b)

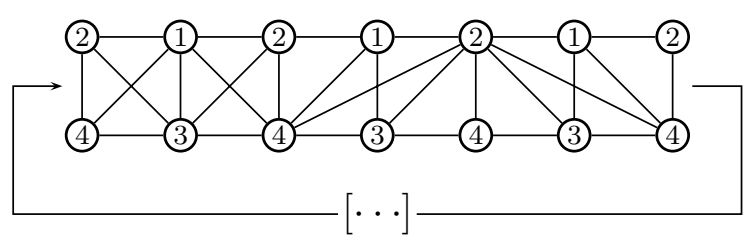

Figure 6: Case 1.

Case 2: The number of $i$-triangles is odd, and the number of e-triangles is even in $G_{Q}^{*}$. Then, by Lemma $2.1, G_{Q}^{*}$ has $m=2 l+1$ vertices, $l \geq 4$, and we can label the vertices on the external cycle with $v_{1}, v_{2}, \ldots, v_{2 k}$, for some $k \geq 2$, and the vertices on the internal cycle with $v_{2 k+1}, \ldots, v_{m}$, in the clockwise manner; additionally suppose that $\left(v_{m} v_{2 k+1} v_{1}\right)$ is an $i$-triangle. (See Fig. 7.) For any vertex $v$ in $G_{Q}^{*}$, let $N(v)$ denote the open neighborhood of $v$, that is, $N(v)=\left\{x \in V\left(G_{Q}^{*}\right):\{v, x\} \in E\left(G_{Q}^{*}\right)\right\}$. Now, if we replace vertex $v_{m}$ with two vertices $v_{m}^{\prime}$ and $v_{m}^{\prime \prime}$ in such a way that $v_{m}^{\prime}$ is adjacent to all vertices in $N\left(v_{m}\right) \backslash\left\{v_{2 k+1}\right\}$ and $v_{m}^{\prime \prime}$ is only adjacent to $v_{1}$ and $v_{2 k+1}$, then one can easily check that the function $c$ defined below is a legal 4-coloring of the new graph $G_{Q}^{*}$.

$$
c\left(v_{i}\right)= \begin{cases}1 & \text { if }(i \leq 2 k) \text { and }(i \bmod 2=1), \\ 2 & \text { if }(i \leq 2 k) \text { and }(i \bmod 2=0), \\ 3 & \text { if }((2 k<i<2 l+1=m) \text { and }(i \bmod 2=1)) \text { or }\left(v_{i}=v_{m}^{\prime}\right), \\ 4 & \text { if }((2 k<i<2 l+1=m) \text { and }(i \bmod 2=0)) \text { or }\left(v_{i}=v_{m}^{\prime \prime}\right) .\end{cases}
$$

Case 3: The number of $i$-triangles is even, and the number of e-triangles is odd in $G_{Q}^{*}$. This case can be proved in the same manner as above: we only have to consider an endpoint of the external edge of an $e$-triangle.

Case 4: The number of $i$-triangles is odd, and the number of e-triangles is odd. Then there is either an $i$-triangle $t_{i}$ or an $e$-triangle $t_{e}$ that shares an edge with a complete subgraph graph of order 4 (a balanced quadrilateral) — without loss of generality we can assume it to be $t_{i}$. Next, let the vertices on the external cycle be labeled with $v_{1}, v_{2}, \ldots, v_{2 k+1}$, for some $k \geq 2$, and the vertices on the internal cycle be labeled with $v_{2 k+2}, \ldots, v_{m}$, in the clockwise manner, in such a way that $i$-triangle $t_{i}$ is labeled $\left(v_{m} v_{2 k+2} v_{1}\right)$ and vertices $v_{1}, v_{2}, v_{2 k+2}$, and $v_{2 k+3}$ induce a complete subgraph of order 4 in $G_{Q}^{*}$. (See Fig. 8.) Notice that $m$ is even. Now, if we replace vertex $v_{1}$ with two vertices $v_{1}^{\prime}$ and $v_{1}^{\prime \prime}$ so that $v_{1}^{\prime}$ is adjacent to all vertices in $N\left(v_{1}\right) \backslash\left\{v_{2}, v_{2 k+3}\right\}$ and $v_{1}^{\prime \prime}$ is adjacent to $v_{2}, v_{2 k+2}$ and $v_{2 k+3}$, 
(a)

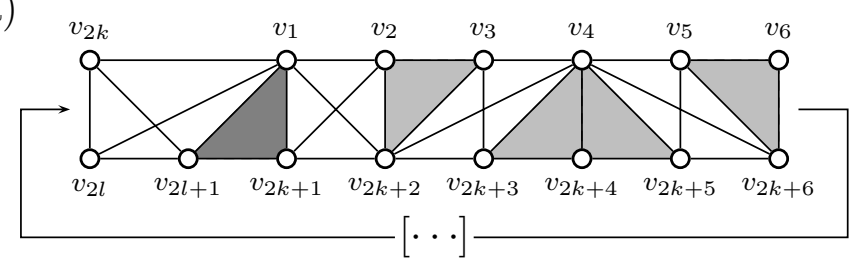

(b)

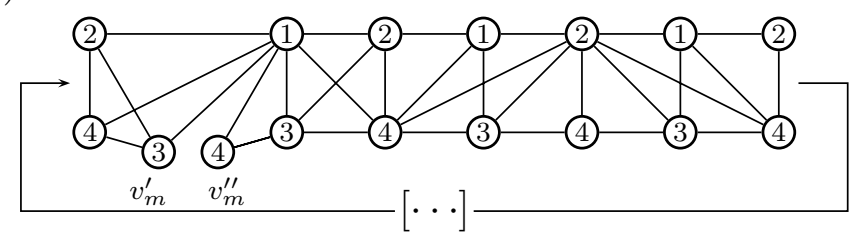

Figure 7: Case 2.

(a)

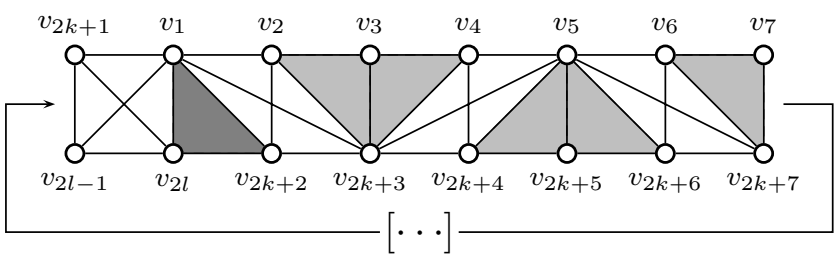

(b)

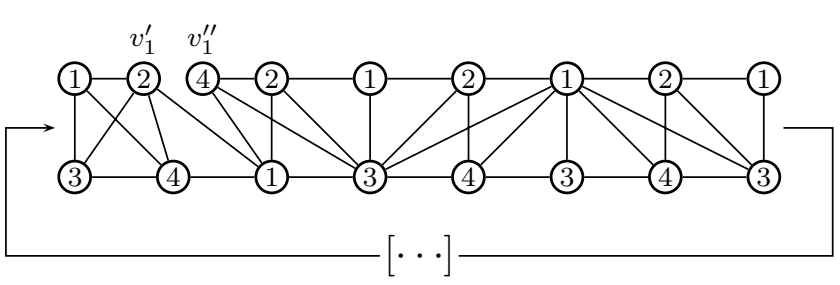

Figure 8: Case 4.

then the function $c$ defined below is a legal 4-coloring of the new graph $G_{Q}^{*}$.

$$
c\left(v_{i}\right)= \begin{cases}1 & \text { if }((2 \leq i \leq 2 k+1) \text { and }(i \bmod 2=1)) \text { or }\left(v_{i}=v_{2 k+2}\right) \\ 2 & \text { if }((2 \leq i \leq 2 k+1) \text { and }(i \bmod 2=0)) \text { or }\left(v_{i}=v_{1}^{\prime}\right) \\ 3 & \text { if }(2 k+3 \leq i \leq m) \text { and }(i \bmod 2=1), \\ 4 & \text { if }((2 k+3 \leq i \leq m) \text { and }(i \bmod 2=0)) \text { or }\left(v_{i}=v_{1}^{\prime \prime}\right)\end{cases}
$$

\subsection{Alternate proof of Aggarwal's Theorem}

The main difficulty of Aggarwal's Theorem lies in showing that $\left\lfloor\frac{n}{4}\right\rfloor$ vertex guards always suffice to cover a one-hole orthogonal polygon with $n$ vertices, as the two-hole case is a 


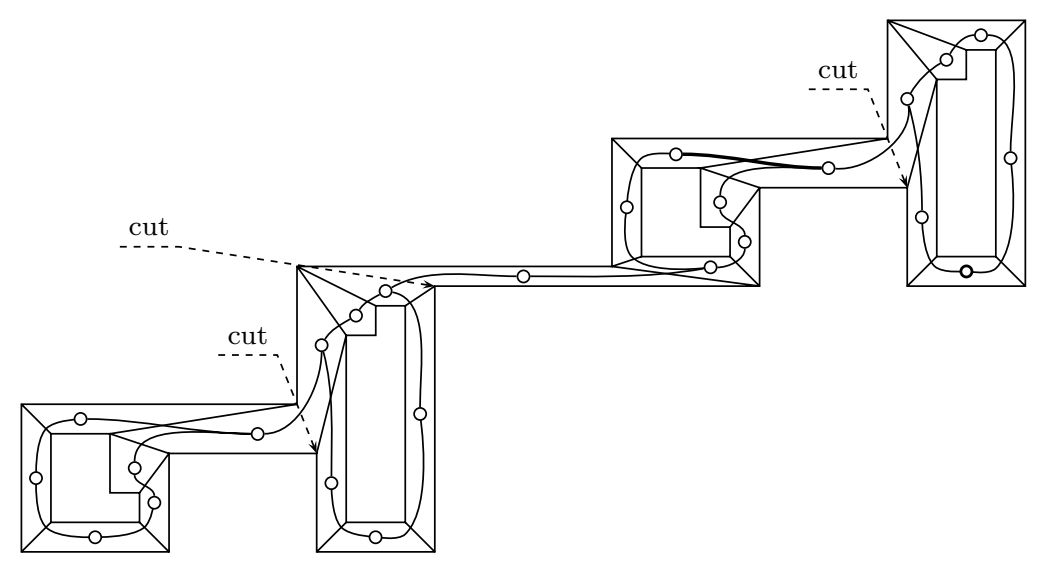

Figure 9: The dual graph of a quadrilateralization of the polygon from Fig. 2.

simple extension of the one-hole theorem [1]. However, bearing in mind Claim 2.2 and Lemma 2.3, we can greatly simplify this step.

Theorem 2.4 [1] Let $P$ be an n-vertex orthogonal polygon with one hole. Then $\left\lfloor\frac{n}{4}\right\rfloor$ vertex guards are occasionally necessary and always sufficient to see the entire interior of $P$.

Proof. By Claim 2.2 and Lemma 2.3, after splitting at most one vertex into two, the graph $G_{Q}$ can be 4-colored. Thus the least frequently used color — say blue — is used at most $\left\lfloor\frac{n+1}{4}\right\rfloor$ times. As any convex quadrilateral has all four colors on its vertices, guards located at the blue vertices will cover the whole polygon. Because an orthogonal polygon must have an even number of vertices, the conclusion follows.

\section{$3 \quad$ Arbitrary number of holes}

For the case of more holes, let us first consider the dual graph of the quadrilateralization of the polygon from Fig. 2. It consists of four cycles connected by single edges, as illustrated in Fig. 9. Cutting along three diagonals of the quadrilateralization results in four one-hole polygons with quadrilateralizations $Q_{i}$ and their quadrilateralization graphs $G_{Q_{i}}$, $i=1,2,3,4$, respectively. These polygons are usually not orthogonal, but it is easy to see that for each $i=1,2,3,4$, there exists a one-hole orthogonal polygon $P^{i}$ with a quadrilateralization graph $G_{Q}^{i}$ isomorphic to graph $G_{Q_{i}}$. Thus by Lemma 2.3, all graphs $G_{Q_{i}}$, $i=1,2,3,4$, are 4 -colorable, after splitting at most four vertices in total. Now, we can reunite the polygons, thus getting a 4-coloring of graph $G_{Q}$ with at most four splits (we can recolor graphs $G_{Q_{i}}, i=1,2,3,4$, if necessary). The least frequently used color will be used not more than $\left\lfloor\frac{n+4}{4}\right\rfloor$ times. The same argument can be applied to any orthogonal polygon with $h$ holes, but only if the dual graph consists of $h$ cycles connected by trees: cut the polygon along $h-1$ diagonals of the quadrilateralization to get $h$ "orthogonal" polygons with one hole, 4-color all graphs $G_{Q_{i}}, i=1, \ldots, h$, and reunite the polygons. Placing guards at the vertices assigned to the least frequently used color (at most $\left\lfloor\frac{n+h}{4}\right\rfloor$ 

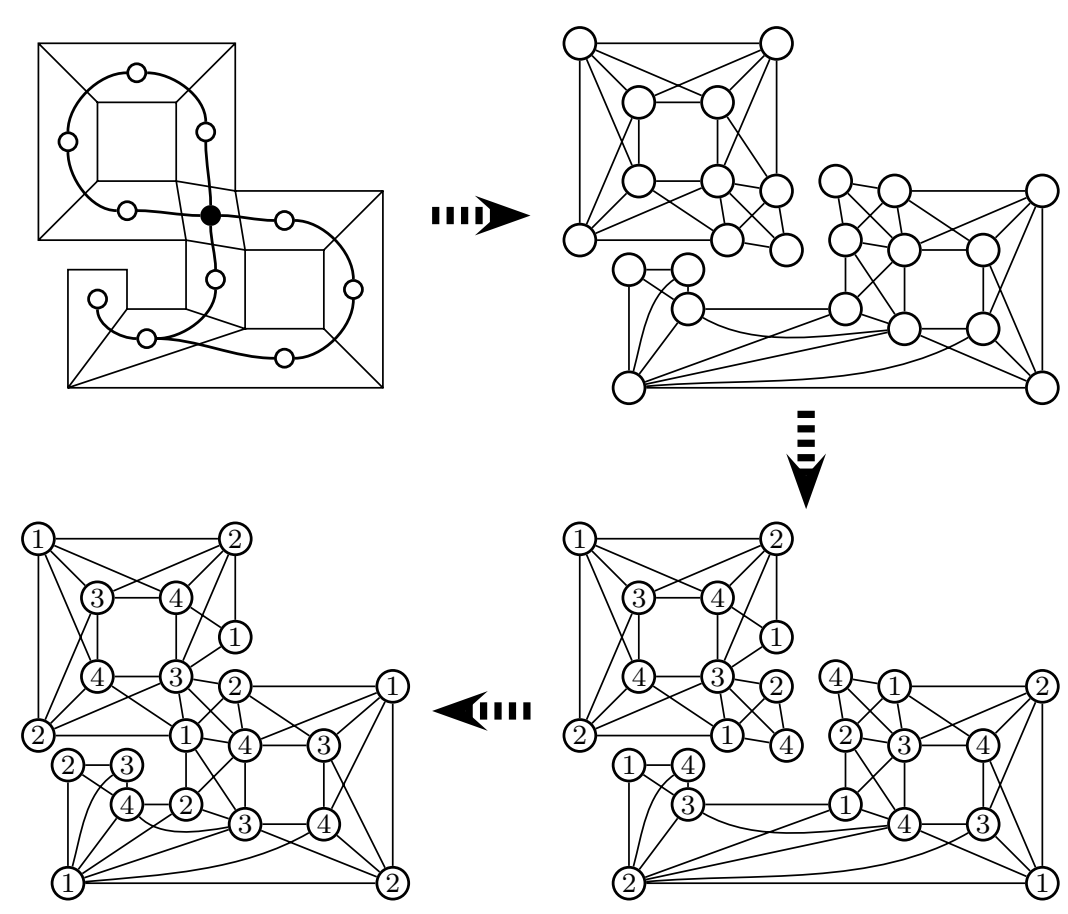

Figure 10: Two cycles of the dual graph have only one vertex in common.

times) will cover the interior of $P$. This is the case when no two cycles in the dual graph have a vertex in common.

Now, let us consider the dual graph of the quadrilateralization of the polygon in Fig. 10 . It has two cycles with only one vertex $v$ in common. We can also apply Lemma 2.3 to this case: we have to split the graph and duplicate vertex $v$, thus getting quadrilateralization graphs $G_{Q_{1}}$ and $G_{Q_{2}}$, respectively. We can obtain a 4-coloring of the graph $G_{Q}$ from 4-colorings of graphs $G_{Q_{1}}$ and $G_{Q_{2}}$, and the least frequently used color must be used at most $\left\lfloor\frac{n+2}{4}\right\rfloor$ times. Furthermore, we can extend this observation to any number of holes only if any two cycles of the dual graph have only one vertex in common, and any such vertex is a cut-vertex. More precisely, suppose that the dual graph of the quadrilateralization of an orthogonal polygon consists of $h$ cycles, any two of them having only one vertex in common, and any such vertex is a cut-vertex - let $v_{1}, \ldots, v_{h-1}$ be these cut-vertices. We have to split the dual graph and duplicate vertices $v_{1}, \ldots, v_{h-1}$, thus getting $h$ cycles and their corresponding graphs $G_{Q_{1}}, \ldots, G_{Q_{h-1}}$, each of them isomorphic to a quadrilateralization graph of a one-hole orthogonal polygon, and each of them 4colorable after splitting at most one vertex in each graph (by Lemma 2.3). Now, we can obtain a 4-coloring of graph $G_{Q}$ by reuniting and possibly recoloring 4-colorings of graphs $G_{Q_{i}} i=1, \ldots, h$ (compare Fig 10). As we have split at most $h$ vertices in total, the least frequently used color is used at most $\left\lfloor\frac{n+h}{4}\right\rfloor$ times. Hence by combining all the above observations, the thesis of Theorem 1.5 follows. 


\section{Final remarks}

The general case of the sufficiency of $\left\lfloor\frac{n+h}{4}\right\rfloor$ vertex guards for an arbitrary $n$-vertex orthogonal polygon with $h$ holes remains open. However, the result of Theorem 1.5 suggests that a potential proof could be based upon vertices (quadrilaterals) which belong to at least two fundamental cycles in the dual graph of a quadrilateralization.

\section{References}

[1] A. Aggarwal, The Art Gallery Theorem: Its Variations, Applications, and Algorithmic Aspects, Ph.D. Thesis, The Johns Hopkins University 1984.

[2] V. Chvátal, A combinatorial theorem in plane geometry, J. Combin. Theory Ser. B 18 (1975), 39-41.

[3] S. Fisk, A short proof of Chvátal's watchman theorem, J. Combin. Theory Ser. B 24 (1978), 374 .

[4] F. Hoffmann, The Art Gallery Problem for rectilinear polygons with holes, Technical Report B 94-22, Freie Universität Berlin 1994.

[5] F. Hoffmann, K. Kriegel, A graph coloring result and its consequences for polygon guarding problems, SIAM J. Discrete Mathematics 9(2) (1996), 210-224.

[6] J. Kahn, M. Klawe, D. Kleitman, Traditional galleries require fewer watchmen, SIAM J. Algebraic and Discrete Methods 4 (1983), 194-206.

[7] J. O'Rourke, Art Gallery Theorems and Algorithms, Oxford University Press 1987.

[8] J. Urrutia, Art Gallery and Illumination Problems, In: Handbook of Computational Geometry, Amsterdam, Elsevier 2000.

[9] P. Żyliński, Placing Guards in Art Galleries by Graph Coloring, In: Graph Colorings (Contemporary Mathematics 352), Ann Arbor, AMS 2004. 